DERECHO Y POLÍTICAS AMBIENTALES EN LA RIOJA (SEGUNDO SEMESTRE 2017)

\author{
MARÍA DEL CARMEN BOLAÑO PIÑEIRO
}

Profesora de Derecho Administrativo

Universidad del País Vasco/ Euskal Herriko Uniberstsitatea 
Sumario: 1. Panorámica general. 2. Ley para la protección del medio ambiente en La Rioja. 3. Incendios. 3.1. Plan Especial de Protección Civil de Emergencia por Incendios Forestales en la Comunidad Autónoma de La Rioja. 3.2. Bases reguladoras de las subvenciones para reforestaciones y creación de superficies forestales. 4. Áreas naturales singulares. 5. Especies amenazadas. 6. Rutas verdes. 7. Caza. 7.1. Regulación de la caza mayor en batida, caza a rececho y caza menor en la Reserva Regional de Caza Cameros Demanda y en los cotos sociales de caza de la Comunidad Autónoma de La Rioja en la temporada 2017-2018. 7.2. Limitaciones y períodos hábiles de caza en la Comunidad Autónoma de La Rioja para la temporada 2017/2018. 8. Pesca. 9. Lista de normas.

\section{PANORÁMICA GENERAL}

Son varias y variadas las normas reglamentarias dictadas en materia medioambiental en la Comunidad Autónoma de La Rioja en el período entre mayo y octubre de 2017. Algunas se corresponden con las que se aprueban cada año en el semestre objeto de análisis, siendo este el caso de las dictadas en la materia de caza. Además, deben mencionarse otras normas aprobadas en dicho intervalo de tiempo, como la norma que declara como áreas naturales singulares determinados espacios y las regula, la norma que establece las bases para la otorgación de ayudas para la protección de las zonas de nidificación del sisón, la disposición que regula dos rutas verdes en la Comunidad Autónoma de La Rioja o el Plan Especial de Protección Civil de Emergencia por Incendios Forestales en la Comunidad Autónoma de La Rioja. Asimismo, en lo que se refiere a las normas con rango de ley, se ha aprobado la que sin duda es la norma más destacable de este periodo: la Ley 6/2017, de 8 de mayo, de Protección del Medio Ambiente de la Comunidad Autónoma de La Rioja, norma de la cual se dará cuenta en esta crónica de forma especial.

A continuación, se procederá a comentar las normas mencionadas.

\section{LEY PARA LA PROTECCIÓN DEL MEDIO AMBIENTE EN LA RIOJA}

Como se adelantaba en la introducción, en el periodo objeto de compilación de esta crónica se ha aprobado la Ley 6/2017, de 8 de mayo, de Protección del Medio Ambiente de la Comunidad Autónoma de La Rioja, que deroga la anterior Ley 5/2002, de 8 de octubre, de Protección del Medio Ambiente en La Rioja. Por razones obvias, no se va a describir detalladamente la norma, sino que tan solo se van a destacar de la misma algunos aspectos de interés como 
las razones de su aprobación, su estructura y las principales diferencias respecto de la norma derogada.

La razón principal que se esgrime en la exposición de motivos para la aprobación de la ley, y la derogación de la anterior, es la modificación de las principales normas básicas estatales en materia medioambiental, lo que habría supuesto el desplazamiento de la normativa riojana en algunos aspectos. En concreto, se mencionan específicamente, entre otras, la Ley 21/2013, de 9 de diciembre, de Evaluación Ambiental, la Ley 5/2013, de 11 de junio, por la que se modifican la Ley 16/2002, de 1 de julio, de prevención y control integrados de la contaminación y la Ley 22/2011, de 28 de julio, de residuos y suelos contaminados, y la Ley 20/2013, de 9 de diciembre, de garantía de la unidad de mercado Ley de Garantías de Unidad de Mercado.

En lo que se refiere a la estructura de la norma debe señalarse que pocos son los cambios en la misma, más allá de alguna alteración en la posición en el articulado o algún añadido como consecuencia de la aprobación de las normas básicas estatales mencionadas. La Ley 6/2017 consta de un título preliminar referido a las disposiciones generales, al que se le inserta una escueta regulación sobre información y participación ciudadana en materia de medio ambiente y la regulación básica del Consejo Asesor de Medio Ambiente de la Comunidad Autónoma de La Rioja. El título primero, que regula la intervención administrativa, se divide en cinco capítulos referidos respectivamente a las generalidades, a la evaluación ambiental, a la autorización ambiental integrada de proyectos y actividades, a las licencias ambientales y a la declaración responsable de apertura. El título segundo regula los instrumentos de actuación medioambiental y se divide en cuatro capítulos referidos, en el siguiente orden, a los planes y programas de protección ambiental, a los sistemas de gestión y auditorías ambientales, a los distintivos de garantía de calidad ambiental y a los instrumentos económicos y de gestión. El título tercero, denominado de «disciplina ambiental», se divide en tres capítulos relativos, respectivamente, a la inspección, control y vigilancia, al régimen sancionador y a los medios de ejecución.

Asimismo, la ley consta de: 1) una disposición adicional relativa a los acuerdos voluntarios; 2) tres disposiciones transitorias referidas, respectivamente, a la 
caducidad de las licencias ambientales vigentes, a la aplicabilidad del Anexo V del Decreto 62/2006, de 10 de noviembre, por el que se aprueba el Reglamento de Desarrollo del Título I, «Intervención Administrativa», de la Ley 5/2002, mientras no se apruebe el reglamento de desarrollo de la Ley 6/2017, y a la evaluación ambiental estratégica del planeamiento urbanístico e instrumentos de ordenación del territorio de La Rioja; 3) una disposición derogatoria; y 4) dos disposiciones finales, una previendo la habilitación de desarrollo de la norma y otra estableciendo su entrada en vigor a los veinte días siguientes de la publicación en el Boletín Oficial de La Rioja .

Las principales diferencias de la Ley 6/2017 con la norma derogada se refieren a la inclusión de las previsiones de las normas básicas estatales en la norma autonómica. Así, se señala la simplificación administrativa como una de las más importantes modificaciones, siendo esta simplificación aplicación directa de la Directiva de Servicios y de su norma de transposición. Además, llama la atención la remisión en bloque a la normativa básica estatal en materia de participación, acceso a la información y a la justicia en materia ambiental. En cuanto a los instrumentos de intervención administrativa, se regula con mayor detalle la licencia ambiental y la declaración responsable de apertura, suponiendo esta última la mayor innovación normativa de la norma a juicio del legislador. Por medio de esta figura se saca del control previo administrativo el ejercicio de algunas actividades que se entiende que pueden tener una menor incidencia negativa en el medio ambiente, conllevando la supresión de la licencia de apertura. En cuanto a sistemas de gestión y auditoría medioambiental se reconoce además de la ISO 14.000 la EMAS, el sistema de gestión y auditoría medioambientales de la Unión Europea.

\section{INCENDIOS}

En materia de incendios dos son las normas reglamentarias que se han aprobado en este periodo. Por un lado, la norma cuya aprobación en este intervalo de tiempo ya es costumbre y que establece las bases reguladoras de las subvenciones para reforestaciones y creación de superficies forestales y, por otro, el Plan Especial de Protección Civil de Emergencia por Incendios 
Forestales en la Comunidad Autónoma de La Rioja, que deroga el anterior que data del año 2005. Se comenzará por este último.

\subsection{Plan Especial de Protección Civil de Emergencia por Incendios Forestales en la Comunidad Autónoma de La Rioja}

En el periodo de este segundo semestre, se aprobó el Decreto 31/2017, de 30 de junio, por el que se aprueba el Plan Especial de Protección Civil de Emergencia por Incendios Forestales en la Comunidad Autónoma de La Rioja (INFOCAR), que deroga el Decreto 58/2005, de 9 de septiembre, por el que se aprueba el Plan Especial de Protección Civil de Emergencia por Incendios Forestales en la Comunidad Autónoma de La Rioja (INFOCAR). La norma, que es desarrollo de las Leyes 17/2015, de 9 de julio, del Sistema Nacional de Protección Civil y 1/2011, de 7 de febrero, de Protección Civil y Atención de Emergencias de La Rioja, y el Real Decreto 893/2013, de 15 de noviembre por el que se aprueba la Directriz básica de planificación de protección civil de emergencia por incendios forestales, recoge un extenso plan de noventa y una páginas -más del doble que el plan anterior-. Las razones de su aprobación son las obvias: la amenaza que constituyen los incendios forestales tanto para el medio ambiente como para el ser humano y la necesidad de planificar su prevención. Asimismo, se señala la necesidad de adoptar el plan a las nuevas necesidades y a las nuevas técnicas.

El decreto se estructura en un artículo, una disposición adicional, una disposición derogatoria, una disposición final y el anexo que constituye el plan en sí mismo. El plan, que en estructura poco cambia en relación con el plan derogado, se compone de seis capítulos que recogen, respectivamente, los fundamentos, los elementos básicos para la planificación de protección civil por incendios forestales, la estructura, organización y funciones, la operatividad, la implantación y el mantenimiento del plan y el catálogo de medios y recursos. En concreto, este último capítulo sería un añadido al plan derogado. Asimismo, el plan recoge siete anexos al anexo del decreto, en los cuales se establece, por el siguiente orden, el índice de gravedad potencial de un incendio forestal; el análisis de riesgo; los municipios considerados de riesgo de incendio forestal; el catálogo de medios y recursos; la jerarquía del personal 
perteneciente al órgano autonómico encargado de la lucha contra incendios forestales; una serie de especificaciones relativas a los planes de autoprotección por riesgo de incendio forestal de las nuevas edificaciones o instalaciones ubicadas en áreas de interfaz urbano-forestal; y, finalmente, algunas disposiciones relativas a los planes de autoprotección por riesgo de incendio forestal de las instalaciones de acampada. Estos anexos constituyen el grueso de las novedades respecto del plan derogado. En concreto, el contenido de los anexos cuatro a siete no se recogía en el anterior plan. El plan entrará en vigor el 1 de enero de 2018.

\subsection{Bases reguladoras de las subvenciones para reforestaciones $y$ creación de superficies forestales}

También ha sido aprobada la Orden 5/2017, de 2 de mayo, de la Consejería de Agricultura, Ganadería y Medio Ambiente, por la que se modifica la Orden 37/2015, de 16 de septiembre, de la Consejería de Agricultura, Ganadería y Medio Ambiente, por la que se establecen las bases reguladoras de las subvenciones para reforestaciones y creación de superficies forestales. La orden modificada fue descrita en una crónica anterior, razón por la cual para un conocimiento mayor de la misma debe remitirse a aquella.

La modificación se lleva a cabo para ajustar la Orden 37/2015 a la Decisión de ejecución de la Comisión Europea de 30 de enero de 2017, por la cual se aprobó la modificación del PDR2014-2020 de La Rioja a efectos de la concesión de ayudas del FEADER, lo que afectaría a la operación 8.1.1, concretamente a la actuación «Primas de mantenimiento», cuyo período de aplicación máximo se redujo de cinco a tres años, contados a partir de la anualidad siguiente al año en que se certifiquen las plantaciones de las reforestaciones. Asimismo, se adapta la orden a la Ley 39/2015, de 1 de octubre, del Procedimiento Administrativo Común de las Administraciones Públicas. Para ello, se modifican diez preceptos.

\section{4. ÁREAS NATURALES SINGULARS}

En una crónica anterior se dio cuenta de la publicación del acuerdo de inicio del expediente de aprobación del decreto por el que se declaraban como «áreas 
naturales singulares» algunas zonas de la Comunidad Autónoma de La Rioja. En esta crónica se describe el Decreto 36/2017, de 21 de julio, por el que se declaran áreas naturales singulares determinados espacios de la Comunidad Autónoma de La Rioja y se aprueban sus normas de protección, que se aprueba en el periodo objeto de esta crónica, y que se dicta en desarrollo de la Ley 4/2003 de Conservación de los Espacios Naturales de La Rioja.

Este decreto declara áreas singulares las «Zonas húmedas y yasas de La Degollada y El Recuenco», en el término municipal de Calahorra; el «Carrizal de Cofín», en el término municipal de Alfaro; el «Carrascal de Villarroya», en el término municipal de Villarroya; y las «Dolinas de Zenzano» en el término municipal de Lagunilla del Jubera. La exposición de motivos del decreto señala las cualidades de cada uno de los espacios por las cuales se entiende que deben ser declarados áreas naturales singulares, constituyendo en el caso de las dos primeras zonas húmedas de interés, en el caso de la tercera un espacio forestal singular y, el último, se declara así por contener las formaciones más características de relieve kárstico de La Rioja.

El decreto consta de dos artículos, una disposición final única y cuatro extensos anexos. Los artículos se limitan a declarar los señalados espacios áreas naturales singulares y el ámbito de aplicación. La disposición final única señala su entrada en vigor al día siguiente de su publicación (que fue el 26 de julio de 2017). Los anexos, uno por cada área natural singular, establecen con detalle el ámbito territorial, la normativa de protección y regulación de los usos, los objetivos de conservación y directrices para la gestión.

\section{ESPECIES AMENAZADAS}

Por medio de la Orden 14/2017, de 6 de septiembre, de la Consejería de Agricultura, Ganadería y Medio Ambiente, se aprueban las bases reguladoras de las ayudas para la protección de las zonas de nidificación del sisón (Tetrax tetrax) en La Rioja, durante la época de cría. La orden es desarrollo del Decreto 55/2014, de 19 de diciembre, que aprobó el Plan de Gestión de las Aves Esteparias en La Rioja, que recoge la posibilidad de establecer ayudas para el retraso del laboreo de barbecho en torno a los cantaderos de sisón común. El sisón común es un ave esteparia recogida en el Catálogo Español de Especies Amenazadas y en el Catálogo Regional de Especies 
Amenazadas. En la exposición de motivos del decreto se señala como principal amenaza del sisón común la intensificación agraria y, dentro de ella, en concreto, el laboreo de los barbechos en primavera por constituir una limitación para su nidificación.

La orden consta de quince artículos, una disposición final y un anexo que recoge el modelo de solicitud. Se señalan como actuaciones objeto de ayuda la no realización de ningún laboreo ni superficial ni profundo, la no aplicación de herbicidas entre la fecha de localización del cantadero y el 31 de agosto, y la no realización de pastoreo entre la fecha de localización del cantadero y el 30 de junio, debiéndose respetar todas ellas para poder recibir la ayuda. Las personas beneficiarias serán las cultivadoras de fincas en barbecho donde se detecte la existencia de cantaderos de sisón y las personas cultivadoras de fincas de barbecho que estén situadas en su totalidad o en un $50 \%$ en un radio de 300 metros alrededor de los cantaderos. Estas personas potencialmente beneficiarias deberán, asimismo, cumplir con una serie de requisitos más específicos. Además, la norma recoge cómo se llevará a cabo el cómputo de las cuantías de las ayudas, los modos de solicitud y el cumplimiento de plazos, el procedimiento administrativo, las obligaciones de los beneficiarios, las consecuencias del incumplimiento de las obligaciones por las personas beneficiarias y el régimen de compatibilidad, entre otras cuestiones. Finalmente, la disposición final única establece que la fecha de entrada en vigor de la orden será el día siguiente al de su publicación (publicación esta que se llevó a cabo el 11 de septiembre de 2017).

\section{RUTAS VERDES}

El Decreto 37/2017, de 21 de julio declara «Rutas Verdes» la vía romana del Iregua y el camino verde del Alhama, siendo este decreto desarrollo de la Ley 5/2003, de 26 de marzo, reguladora de la Red de Itinerarios Verdes de La Rioja. La creación de una Red de Itinerarios Verdes tiene como objetivo la reutilización de terrenos o infraestructuras ferroviarias en desuso, acondicionándolas para el uso público. Asimismo, se crea la posibilidad de declarar con la denominación de Rutas Verdes otras zonas de similar uso aunque no tengan un origen ferroviario. 
Las labores de acondicionamiento de los senderos denominados «Vía Romana de Iregua», que comunica de forma peatonal Viguera con el Puerto de Piqueras, y el "Camino Verde de del Alhama», que une el Balneario de la Albotea con Aguilar del Río Alhama, llevan a su declaración de Rutas Verdes por medio del mencionado decreto. Entre las razones para su declaración se señala, expresamente, la demanda de la sociedad del disfrute de un turismo de naturaleza respetuoso con el medio ambiente, indicando el atino de los itinerarios verdes como medio para promocionarlo.

El Decreto 37/2017 consta de dos artículos, en los cuales se declaran las Rutas Verdes y se señala la competencia de la Comunidad Autónoma de La Rioja de gestión de las infraestructuras de la Red de Itinerarios Verdes, siendo estos bienes de dominio y de uso públicos. Asimismo, recoge una disposición derogatoria, que deroga en bloque todas las disposiciones de igual o inferior rango que se opongan a lo dispuesto en el decreto, y dos disposiciones finales, habilitando el desarrollo reglamentario y disponiendo su entrada en vigor al día siguiente de su publicación (que fue el 26 de julio de 2017). Finalmente, el decreto incluye un extenso anexo señalando respecto de ambas rutas sus denominaciones, la descripción de sus trazados, los términos municipales que atraviesan, las características generales de los terrenos que recorren, la distancia total, las zonas e instalaciones afectas a su servicio de forma permanente y el régimen de uso de las infraestructuras.

\section{CAZA}

\subsection{Regulación de la caza mayor en batida, caza a rececho y caza menor} en la reserva regional de caza cameros demanda y en los cotos sociales de caza de la Comunidad Autónoma de La Rioja en la temporada 20172018

La Orden 11/2017, de 27 de junio, de la Consejería de Agricultura, Ganadería y Medio Ambiente, por la que se establecen las normas para la caza mayor en batida, caza en rececho y caza menor en la Reserva Regional de Caza de La Rioja, Cameros-Demanda y en los Cotos Sociales de Caza de la Comunidad Autónoma de La Rioja durante la temporada 2017-2018, deroga expresamente 
la Orden 16/2016, de 23 de junio que regulaba la campaña anterior y que fue recogida en la crónica del segundo semestre de 2016.

Tal y como se señala en la exposición de motivos, el fin de la orden no es otro que evitar los aprovechamientos abusivos e implantar un sistema de expedición de permisos que garantice a los cazadores el acceso libre a dichos terrenos en condiciones de equidad, transparencia y a un precio asequible. En esta orden, que no se recogen cambios reseñables respecto de la anterior, se establecen las normas relativas a la organización de batidas, las fechas, las condiciones para la realización de las batidas por tipos, las cuotas de entrada y las cuotas complementarias, el procedimiento para solicitar la organización de batidas, las obligaciones de cazadores y ojeadores y la utilización de perros. También se prevén las normas relativas a la caza menor. En cuanto al régimen sancionador, la norma remite al establecido en la Ley 9/1998, de 2 de julio, de Caza de La Rioja.

La orden entró en vigor al día siguiente de su publicación, publicación que fue el 30 de junio de 2017.

\subsection{Limitaciones y períodos hábiles de caza en la Comunidad Autónoma de La Rioja para la temporada 2016/2017}

La Orden 12/2017, de 19 de julio, de la Consejería de Agricultura, Ganadería y Medio Ambiente, por la que se fijan las limitaciones y períodos hábiles de caza en la Comunidad Autónoma de La Rioja para la temporada cinegética 20172018, que deroga la Orden 15/2016, de 23 de junio, que regulaba la campaña cinegética anterior, establece al igual que la norma derogada la relación de especies cazables y la prohibición general de dar muerte, dañar, molestar o inquietar al resto de especies, sin recoger cambios reseñables respecto de la orden derogada. Nótese que esta última fue brevemente descrita en la segunda crónica del año 2016.

Las especies cazables continúan clasificándose siguiendo los mismos criterios, siendo estos los siguientes: 1) caza menor, en referencia tanto a aves en general como a aves acuáticas; b) caza mayor, que comprende animales como el jabalí, el ciervo, el corzo y el lobo; y c) las denominadas "especies prioritarias", que presentan un nivel poblacional anormal, de las cuales pueden 
destacarse, principalmente, la liebre y la perdiz. Asimismo, la norma se remite a la Ley 9/1998, de 2 de julio, de Caza, que define los terrenos no cinegéticos y establece otras zonas prohibidas para la caza por razón del sitio —en concreto, se hace especial referencia a los lugares donde se encuentran las colonias de cría de buitre leonado entre el 1 de enero y el segundo domingo de septiembre-. Finalmente, la Orden prevé los períodos de caza teniendo en cuenta las especies cazables y sus limitaciones, así como los métodos para el control de especies predadoras, entre otras cuestiones relacionadas.

La norma se publicó el 21 de julio de 2017 y entró en vigor al día siguiente.

8. Pesca

La única norma aprobada en materia de pesca en este periodo es la Orden 8/2017, de 17 de mayo, de la Consejería de Agricultura, Ganadería y Medio Ambiente, que modifica la Orden 1/2017, de 4 de enero, de la Consejería de Agricultura, Ganadería y Medio Ambiente, por la que se fijan los periodos hábiles de pesca y normas relacionadas con la misma en aguas de la Comunidad Autónoma de La Rioja durante el año 2017. La orden modificada fue descrita en la crónica anterior.

Las dos modificaciones se realizan en el Anexo I. Por un lado, se señalan los días pescables atendiendo al tipo de pesca en el Coto de Pesca Intensiva de Anguciana incluido en el subapartado a.1) Cotos de Pesca del apartado A) Cuenca de los ríos Oja-Tirón. En concreto, se señala que en esa zona la pesca tradicional se podrá realizar los miércoles, jueves, viernes, sábados, domingos y festivos y la pesca sin muerte los lunes no festivos. Por otro lado, en relación con el Coto de Pesca de Peroblasco -incluido en el subapartado e.1) Cotos de Pesca del apartado A) Cuenca de los ríos Cidacos y Alhama-, se señala que permanecerá cerrado teniendo en cuenta que el caudal circulante es inviable para permitir un aprovechamiento del espacio de pesca.

Estas modificaciones entraron en vigor el mismo día de su publicación en el Boletín Oficial de La Rioja, publicación que se llevó a cabo el 19 de mayo de 2017.

\section{LISTA DE NORMAS}


Orden 14/2017, de 6 de septiembre, de la Consejería de Agricultura, Ganadería y Medio Ambiente, por la que se aprueban las bases reguladoras de las ayudas para la protección de las zonas de nidificación del sisón (Tetrax tetrax) en La Rioja, durante la época de cría.

Decreto $37 / 2017$, de 21 de julio, por el que se declaran rutas verdes la vía romana del Iregua y el camino verde del Alhama

Decreto 36/2017, de 21 de julio, por el que se declaran áreas naturales singulares determinados espacios de la Comunidad Autónoma de La Rioja y se aprueban sus normas de protección

Orden 12/2017, de 19 de julio, de la Consejería de Agricultura, Ganadería y Medio Ambiente, por la que se fijan las limitaciones y períodos hábiles de caza en la Comunidad Autónoma de La Rioja para la temporada cinegética 20172018

Decreto 31/2017, de 30 de junio, por el que se aprueba el Plan Especial de Protección Civil de Emergencia por Incendios Forestales en la Comunidad Autónoma de La Rioja (INFOCAR)

Orden 11/2017, de 27 de junio, de la Consejería de Agricultura, Ganadería y Medio Ambiente, por la que se establecen las normas para la caza mayor en batida, caza en rececho y caza menor en la Reserva Regional de Caza de La Rioja, Cameros-Demanda y en los Cotos Sociales de Caza de la Comunidad Autónoma de La Rioja durante la temporada 2017-2018

Orden 8/2017, de 17 de mayo, de la Consejería de Agricultura, Ganadería y Medio Ambiente, por la que se modifica la Orden 1/2017, de 4 de enero, de la Consejería de Agricultura, Ganadería y Medio Ambiente, por la que se fijan los periodos hábiles de pesca y normas relacionadas con la misma en aguas de la Comunidad Autónoma de La Rioja durante el año 2017.

Ley 6/2017, de 8 de mayo, de Protección del Medio Ambiente de la Comunidad Autónoma de La Rioja.

Orden 5/2017, de 2 de mayo, de la Consejería de Agricultura, Ganadería y Medio Ambiente, por la que se modifica la Orden 37/2015, de 16 de septiembre, de la Consejería de Agricultura, Ganadería y Medio Ambiente, por 
la que se establecen las bases reguladoras de las subvenciones para reforestaciones y creación de superficies forestales 